\title{
Experimental limits on the proton life-time from the neutrino experiments with heavy water
}

\author{
V.I. Tretyak and Yu.G. Zdesenko \\ Institute for Nuclear Research, MSP 03680 Kiev, Ukraine
}

\begin{abstract}
Experimental data on the number of neutrons born in the heavy water targets of the large neutrino detectors are used to set the limit on the proton life-time independently on decay mode through the reaction $d \rightarrow n+$ ? . The best up-to-date limit $\tau_{p}>4 \times 10^{23}$ yr with $95 \%$ C.L. is derived from the measurements with $\mathrm{D}_{2} \mathrm{O}$ target (mass $267 \mathrm{~kg}$ ) installed near the Bugey reactor. This value can be improved by six orders of magnitude with future data accumulated with the SNO detector containing $1000 \mathrm{t}$ of $\mathrm{D}_{2} \mathrm{O}$.
\end{abstract}

PACS: 14.20.D; 24.80.+y; 25.40.-h

Keywords: Proton life-time; Neutrino detectors; Deuteron

\section{Introduction}

The baryon $(B)$ and lepton $(L)$ numbers are absolutely conserved in the Standard Model (SM). However, many extensions of the SM, in particular, grand unified theories incorporate $B$ and $L$ violating interactions, since in the modern gauge theories conservation of baryon (lepton) charge is considered as approximate law due to absence of any underlying symmetry principle behind it, unlike the gauge invariance in electrodynamics which guarantees the massless of photon and absolute conservation of the electric charge. Therefore, it is quite natural to suppose the decay of protons and neutrons bounded in nuclei. The processes with $\Delta B=1, \Delta B=2, \Delta(B-L)=0, \Delta(B-L)=2$ have been discussed in literature (see e.g. [1, 2, 3] and references therein), while the disappearance of nucleons (or decay into "nothing") has been addressed in connection with possible existence of extra dimensions [4, 国, 苜].

Stimulated by theoretical predictions, nucleon instability has been searched for in many underground experiments with the help of massive detectors such as IMB, Fréjus, Kamiokande, SuperKamiokande and others (for experimental activity see [3, 7, 8] and refs. therein). About 90 decay modes have been investigated; however, no evidence for the nucleons decay has been found. A complete summary of the experimental results is given in the Review of Particle Physics [9]. For the modes in which the nucleon decays to particles strongly or electromagnetically interacting in the detector's sensitive volume, the obtained life-time limits are in the range of $10^{30}-10^{33} \mathrm{yr}$, while for decays to only weakly interacting products (neutrinos) the bounds are up to 10 orders of magnitude lower [9, [10]. However, because it is not known a priori which mode of proton decay (from 90 ones listed in [9]) is preferable, the limits on the proton decay independent on the channel are very important. 
Three approaches were used to establish such limits:

(1) In ref. [11 the bound $\tau(p \rightarrow$ ? $)>1.3 \times 10^{23}$ yr was determined] on the basis of the limit for the branching ratio of ${ }^{232} \mathrm{Th}$ spontaneous fission $\beta_{S F}$. It was assumed that the parent ${ }^{232} \mathrm{Th}$ nucleus is destroyed by the strongly or electromagnetically interacting particles emitted in the proton decay or, in case of proton's disappearance (or $p$ decay into neutrinos) by the subsequent nuclear deexcitation process. Using the present-day data [12] on the ${ }^{232} \mathrm{Th}$ half-life $T_{1 / 2}=1.405 \times 10^{10} \mathrm{yr}$ and limit $\beta_{S F}<1.8 \times 10^{-9} \%$, we can recalculate the value of 11] as $\tau(p \rightarrow$ ? $)>1.0 \times 10^{23} \mathrm{yr}$.

(2) In ref. 113 the limit $\tau\left(p \rightarrow\right.$ ?) $>3 \times 10^{23} \mathrm{yr}$ was established by searching for neutrons born in liquid scintillator, enriched in deuterium, as result of proton decay in deuterium $(d \rightarrow n+$ ? $)$.

(3) In ref. [14 the limit $\tau(p \rightarrow 3 \nu)>7.4 \times 10^{24}$ yr was determined] on the basis of geochemical measurements with Te ore by looking for the possible daughter nuclides $\left({ }^{130} \mathrm{Te}\right.$ $\rightarrow \ldots \rightarrow{ }^{129} \mathrm{Xe}$ ), while in refs. [15, [16] the bound $\tau(p \rightarrow 3 \nu)>1.1 \times 10^{26}$ yr was achieved by the radiochemical measurements with $1710 \mathrm{~kg}$ of potassium acetate $\mathrm{KC}_{2} \mathrm{H}_{3} \mathrm{O}_{2}$ placed deep underground $\left({ }^{39} \mathrm{~K} \rightarrow \ldots . \rightarrow{ }^{37} \mathrm{Ar}\right)$. In the experiments (2) and (3) both the baryon number and the electric charge would be not conserved; nevertheless authors suggested that "experimenter would be wise not to exclude such processes from consideration $a$ priori" [14].

The limits [14, 15, 16] usually are quoted as "independent on channel" [9], however it is evident that they are valid only for the proton decay into invisible channels (or disappearance), in which the parent nucleus is not fully destroyed (like ${ }^{232} \mathrm{Th}$ in the experiment [11]). At the same time, bound on the proton decay from the deuterium disintegration requires the less stringent hypothesis on the stability of daughter nuclear system and, hence, it is less model dependent. Such a limit was established in 1970 [13 and is equal $\tau(p \rightarrow ?)>3 \times 10^{23}$ yr at $68 \%$ C.L. T. This value can be improved by using the data from the modern neutrino experiments with heavy water well shielded against cosmic rays and natural radioactivity. With this aim, in present paper we analyze the measurements of ref. [17] with the $267 \mathrm{~kg} \mathrm{D}_{2} \mathrm{O}$ target installed at Reactor 5 of the Centrale Nucleaire de Bugey (France). Further, we show that obtained limit $\tau(p \rightarrow$ ?) can be highly improved with the SNO (Sudbury Neutrino Observatory) large volume detector [18] developed mainly for the Solar neutrino investigations and containing $1000 \mathrm{t}$ of $\mathrm{D}_{2} \mathrm{O}$.

\section{$2 \quad \mathrm{D}_{2} \mathrm{O}$ experiment at the Bugey reactor}

The experiment [17] was aimed to measure the cross sections for the disintegration of deuteron by low-energy electron antineutrinos from nuclear reactor through reactions $\bar{\nu}_{e}+d \rightarrow \bar{\nu}_{e}+n+p$ (neutral currents) and $\bar{\nu}_{e}+d \rightarrow e^{+}+n+n$ (charged currents). Events were recognized by the neutrons they produced. The detector was located on the depth of 25 meters of water equivalent (mwe) at $18.5 \mathrm{~m}$ distance from the center of

\footnotetext{
${ }^{1}$ We recalculated the value quoted in 11$] \tau\left(N \rightarrow\right.$ ?) $>3 \times 10^{23}$ yr (given for 232 particles: 142 neutrons and 90 protons) for 90 protons which should be taken here into consideration ( $N$ is $p$ or $n$ ).

${ }^{2}$ The value $\tau(N \rightarrow 3 \nu)>1.6 \times 10^{25}$ yr quoted in [14] as given for 52 particles (28 neutrons and 24 protons) was recalculated for 24 protons.

${ }^{3}$ Because ref. [13] is not the source of easy access, and in ref. [9], where this limit is quoted, there is no indication for confidence level, we suppose that it was established with $68 \%$ C.L.
} 
the Reactor 5 core at the Bugey site. The cylindrical target tank, containing $267 \mathrm{~kg}$ of $99.85 \%$ pure $\mathrm{D}_{2} \mathrm{O}$, was surrounded by layers of lead $(10 \mathrm{~cm})$ and cadmium $(1 \mathrm{~mm})$ to absorb thermal neutrons from external surroundings. The tank with $\mathrm{D}_{2} \mathrm{O}$ and $\mathrm{Pb}-\mathrm{Cd}$ shield was inserted in the center of a large liquid scintillator detector (based on mineral oil) which served as (inner) cosmic ray veto detector. Subsequent layer of lead $10 \mathrm{~cm}$ thick was aimed to reduce the flux of external $\gamma$ quanta with energies $E_{\gamma}>2.23 \mathrm{MeV}$ which can photodisintegrate the deuterons creating the background events. However this shielding itself was a significant source of neutrons in the target detector: they were created due to interaction of cosmic rays with $\mathrm{Pb}$. To suppress this background, an additional layer of cosmic ray veto detectors was installed outside the $\mathrm{Pb}$ shielding. The outer veto reduced the neutron background in the target by a factor of near 6 . Neutrons were detected by ${ }^{3} \mathrm{He}$ proportional counters installed in the tank with $\mathrm{D}_{2} \mathrm{O}$ through reaction ${ }^{3} \mathrm{He}+n \rightarrow$ ${ }^{3} \mathrm{H}+p+764 \mathrm{keV}$. Further details on the experiment can be found in [17].

The decay or disappearance of proton bounded in deuterium nucleus, which consists only of proton and neutron, will result in the appearance of free neutron: $d \rightarrow n+$ ? . Thus the proton life-time limit can be estimated on the basis of the neutron rate detected in the $\mathrm{D}_{2} \mathrm{O}$ volume when the reactor is switched off. To calculate the $\lim \tau(p \rightarrow ?)$ value, we use the formula

$$
\lim \tau(p \rightarrow ?)=\varepsilon \times N_{d} \times t / \lim S,
$$

where $\varepsilon$ is the efficiency for the neutron's detection, $N_{d}$ is number of deuterons, $t$ is the time of measurement, and $\lim S$ is the number of proton decays which can be excluded with a given confidence level on the basis of the neutron background (one-neutron events) measured in the experiment. Mean efficiency for single neutrons born isotropically throughout the $\mathrm{D}_{2} \mathrm{O}$ volume was determined as $\varepsilon=0.29 \pm 0.01$ [17]. In $267 \mathrm{~kg}$ of $\mathrm{D}_{2} \mathrm{O}$ there is $N_{d}=1.605 \times 10^{28}$ deuterons. Raw one-neutron rate with the reactor down is equal $25.28 \pm 0.68$ counts per day (cpd) and, corrected for software efficiency $(0.444 \pm 0.005)$, this rate is $57.00 \pm 1.53 \mathrm{cpd}$. For very rough estimate of the $p$ life-time (as the first approximation) we can attribute all neutron events to proton decays and obtain the $\lim S$ value as $59.5 \mathrm{cpd}$ at $95 \%$ C.L. Then, substituting this value in the formula (1) we get limit $\tau(p \rightarrow ?)>2.1 \times 10^{23} \mathrm{yr}$ with $95 \%$ C.L.f However, it is evident that $\tau$ limit derived in this way is very conservative because the dominant part of observed neutron rate has other origins rather than proton decay [20, 21, 22]. On the other hand, if we suppose that all measured neutron events are belonging to background, then the excluded number of neutrons due to possible proton decay will be restricted only by statistical uncertainties in the measured neutron background. Hence, to estimate value of $\lim S$ we can use so called "one (two, three) $\sigma$ approach", in which the excluded number of effect's events is determined simply as square root of the number of background counts multiplied by one (two or three) according to the confidence level chosen $(68 \%, 95 \%$ or $99 \%)$. This method gives us the sensitivity limit of the considered experiment to the proton decay. Applying it we get $\lim S=3 \mathrm{cpd}$ (at $95 \%$ C.L.), which leads to the bound $\tau\left(p \rightarrow\right.$ ?) $>4 \times 10^{24} \mathrm{yr}$.

Therefore, we can argue that, with the probability close to $100 \%$, estimate of $\tau$ limit is within interval $2 \times 10^{23} \mathrm{yr}-4 \times 10^{24} \mathrm{yr}$. In order to fix life-time limit or at least to narrow this interval, it is necessary to determine the contributions of different sources to the total

\footnotetext{
${ }^{4}$ The similar limit $\tau(p \rightarrow ?)>1.9 \times 10^{23}$ yr with $95 \%$ C.L. can be derived from other neutrino deuteron experiment at Krasnoyarsk (Russia) nuclear reactor [19].
} 
Table 1: Main characteristics of the neutrino experiments at nuclear reactor

\begin{tabular}{|l|l|l|l|l|}
\hline Experiment & $\begin{array}{l}\text { Bugey-1995 } \\
{[24]}\end{array}$ & $\begin{array}{l}\text { Bugey-1999 } \\
{[17]}\end{array}$ & $\begin{array}{l}\text { Palo Verde } \\
{[25]}\end{array}$ & $\begin{array}{l}\text { Chooz } \\
{[26]}\end{array}$ \\
\hline Depth & $40 \mathrm{mwe}$ & $25 \mathrm{mwe}$ & $32 \mathrm{mwe}$ & $300 \mathrm{mwe}$ \\
\hline Distance & $15 \mathrm{~m}$ & $18.5 \mathrm{~m}$ & $\approx 0.9 \mathrm{~km}$ & $\approx 1 \mathrm{~km}$ \\
\hline Detector type & $\begin{array}{l}\text { Li-loaded } \\
\text { scintillator }\end{array}$ & $\begin{array}{l}\mathrm{D}_{2} \mathrm{O} \text { target }+ \\
{ }^{3} \mathrm{He} \text { counters }\end{array}$ & $\begin{array}{l}\text { Gd-loaded } \\
\text { scintillator }\end{array}$ & $\begin{array}{l}\text { Gd-loaded } \\
\text { scintillator }\end{array}$ \\
\hline Detector mass & $\approx 0.6 \mathrm{t}$ & $267 \mathrm{~kg}$ & $11 \mathrm{t}$ & $5 \mathrm{t}$ \\
\hline $\begin{array}{l}\text { Neutron } \\
\text { background }\end{array}$ & $\approx 100 \mathrm{cpd} / \mathrm{t}$ & $\approx 100 \mathrm{cpd} / \mathrm{t}$ & $2.2 \mathrm{cpd} / \mathrm{t}$ & $0.24 \mathrm{cpd} / \mathrm{t}$ \\
\hline
\end{tabular}

neutron rate observed. As it was already mentioned, the nature and origins of neutron background in neutrino experiments at nuclear reactors are well known and understood (see, for example, refs. [20, 21, 22, 23]). The main sources are: (i) interaction of cosmic muons (escaped an active veto system) with the detector, passive shield and surrounding materials; (ii) photodisintegration of the deuteron by $\gamma$ quanta (with $E_{\gamma}>2.23 \mathrm{MeV}$ ), originated from the radioactive contamination of the detector materials and shield, as well as from environment pollution; (iii) residual (and non-eliminated by the shield) neutron background at the nuclear reactor site.

Before coming to details, we would like to remind that crucial characteristics of any neutrino experiment at reactor are the depth of its location and distance from the reactor core [20, 21, 22, 23. Let us prove this statement by Table 1 with parameters of the most advanced experiments and by two short citations, from ref. 20]: "A striking difference between the two experiments is the amount of overburden, which may be viewed as the main factor responsible for how the experiments compare on detector design, event rate, and signal-to-background", and from ref. [21]: "... a detector should be located sufficiently deep underground to reduce the flux of cosmic muons - the main source of background in experiments of this type".

It is clear from the Table 1 that neutron background of different experiments is decreased as the depth of their location and distance from the reactor are enlarged. For example, in the Chooz experiment with liquid scintillator the background was reduced roughly by factor 500 as compared with that of Bugey ones [17, 24] because the Chooz set up was placed deep underground (300 mwe) and $\approx 1 \mathrm{~km}$ away from nuclear reactor [26]. We recall that Bugey set ups [17, 24] were located only 25-40 mwe overburden (which allows to remove the hadronic component of cosmic rays but is not enough to reduce the muon flux significantly) and at 15-18 $\mathrm{m}$ distance from the reactor core. Thus, the dominant part of neutron background in [17, 24] is associated with the reactor site and muon flux. Indeed, as it was proved by the detail simulation and careful analysis of neutron background in reactor-off periods of the experiment [24], the $67 \pm 3 \%$ of neutron rate (measured at $15 \mathrm{~m}$ distance) are attributed to known origins (see Table 6 in ref. 24]). On the basis of comparison of different experiments presented in Table 1, and taking into account results of background analysis [24], we can make semi-quantitative and conservative estimation that at least $50 \%$ of one-neutron events measured in [17] are caused by 
the mentioned sources (i) - (iii). Hence, attributing remaining part of one-neutron rate to other unknown background origins, we can accept its value as the excluded number of proton's decays ( $\lim S=30 \mathrm{cpd}$ ). Finally, substituting this number in the formula (1) we obtain

$$
\tau(p \rightarrow ?)>4 \times 10^{23} \text { yr } \quad \text { with } 95 \% \text { C.L. }
$$

which is higher than previous limit [13].

\section{Expected improvements with the SNO solar neu- trino detector}

The Sudbury Neutrino Observatory (SNO) [18] is an unique large Cherenkov detector constructed with an emphasis on the study of Solar neutrinos. The detector, containing $1000 \mathrm{t}$ of $99.917 \%$ isotopically pure heavy water, is located in the INCO Creighton nickel mine near Sudbury, Ontario, on the depth of 2039 m (near 6000 mwe); this reduces the muon flux to 70 muons per day in the detector area. Particular attention is payed to minimization of radioactive backgrounds. Near $7000 \mathrm{t}$ of ultra-pure light water shield the central $\mathrm{D}_{2} \mathrm{O}$ detector from natural radioactivity from the laboratory walls. All components of the detector are made of selected materials with low radioactivity contamination.

Solar neutrinos will be detected through the following reactions with electrons and deuterons: $\nu_{i}+e^{-} \rightarrow \nu_{i}+e^{-}$(elastic scattering; $\left.i=e, \mu, \tau\right), \nu_{e}+d \rightarrow e^{-}+p+p$ (charged current absorption) and $\nu_{i}+d \rightarrow \nu_{i}+n+p$ (neutral current disintegration of deuteron). Near 9600 photomultiplier tubes are used to observe the Cherenkov light produced on the $\mathrm{D}_{2} \mathrm{O}$ volume by high energy products. Neutrons released in $d$ disintegration will be detected by neutron capture on deuterons in pure $\mathrm{D}_{2} \mathrm{O}$, or by capture on ${ }^{35} \mathrm{Cl}$ by dissolving $\mathrm{MgCl}$ salt in the heavy water, or by capture on ${ }^{3} \mathrm{He}$ using proportional counters. Further details can be found in 18$]$.

Extensive Monte Carlo simulations were performed to predict response functions and numbers of expected events due to interaction of the detector with Solar neutrinos, natural radioactivity of various detector components, cosmogenic activities, capture of neutrons, $(\alpha, p \gamma),(\alpha, n \gamma)$ reactions outside the SNO detector, etc. (f.e., see [27]). Expected number of neutrons from all sources in the $\mathrm{D}_{2} \mathrm{O}$ volume is calculated as $\approx 5 \times 10^{3}$ during 1 yr period of exposition, with main contribution from the Solar neutrinos. Efficiency for $n$ detection is equal $83 \%$ for $n$ capture on ${ }^{35} \mathrm{Cl}$ 18, 27].

Using these unique features of the SNO detector (super-low background, large amount of $\mathrm{D}_{2} \mathrm{O}$ and high sensitivity to neutrons), the limit on the proton decay independent on channel can be highly improved. Again for very rough estimate of the $p$ life-time we can conservatively attribute all neutrons in the $\mathrm{D}_{2} \mathrm{O}$ volume to proton decays and accept it as the excluded value $\lim S=5 \times 10^{3}$ counts. Then, substituting in the formula (1) values of efficiency $\varepsilon=0.83$, measuring time $t=1 \mathrm{yr}$, number of deuterons $N_{d}=6 \times 10^{31}$ and $\lim S=5 \times 10^{3}$ counts, we receive

$$
\tau(p \rightarrow ?)>1 \times 10^{28} \mathrm{yr}
$$


which is about five orders of magnitude higher than present-day limit. However this value can be improved further by accounting the neutron events originating from Solar neutrinos and high energy $\gamma$ quanta. Number of neutrons born in the $\mathrm{D}_{2} \mathrm{O}$ volume due to disintegration $\nu_{i}+d \rightarrow \nu_{i}+n+p$ can be estimated independently using the information on the number of Solar neutrino interaction with the detector volume through neutrinoelectron elastic scattering $\nu_{i}+e^{-} \rightarrow \nu_{i}+e^{-}$. Neutrons created by high energy $\gamma$ quanta from natural radioactivity in the detector components can be also calculated if the levels of pollution of all materials are measured firmly. In that case the excluded number of neutrons due to possible proton decay will be restricted only by statistical uncertainties of the measured neutron background, i.e. we can apply "two $\sigma$ approach" again. It gives $\lim S=2 \sqrt{5000}$ with $95 \%$ C.L., thus the corresponding bound on the proton life-time would be equal to

$$
\tau(p \rightarrow ?)>4 \times 10^{29} \text { yr } \quad \text { with } 95 \% \text { C.L. },
$$

which can be considered as the maximal sensitivity of the SNO detector for the proton decay independent on channel.

\section{Conclusion}

The data of the Bugey experiment [17], aimed to measure the cross sections for the deuteron disintegration by antineutrinos from nuclear reactor, were analyzed to set the proton life-time limit. The obtained value $\tau(p \rightarrow ?)>4 \times 10^{23}$ yr at $95 \%$ C.L. is higher than the limit established in the previous experiment 13. With the future data from the SNO Solar neutrino detector (containing 1000 t of heavy water) life-time limit will be improved up to the value $\tau(p \rightarrow ?)>4 \times 10^{29} \mathrm{yr}$, which is, in fact, close to the bounds established for the particular modes of the nucleon decays to charged or strongly interacting particles and would be of a great importance for many extensions of the modern gauge theories.

\section{References}

[1] P. Langacker, Phys. Rep. 71 (1981) 185.

[2] M. Goldhaber, P. Langacker and R. Slansky, Science 210 (1980) 851.

[3] H.V. Klapdor-Kleingrothaus and A. Staudt, Non-accelerator Particle Physics (IoP, Bristol and Philadelphia, 1995).

[4] F.J. Yndurain, Phys. Lett. B 256 (1991) 15.

[5] G. Dvali, G. Gabadadze and G. Senjanovic, hep-ph/9910207.

[6] S.L. Dubovsky, V.A. Rubakov, P.G. Tinyakov, Phys. Rev. D 62 (2000) 105011; JHEP 8 (2000) 041.

[7] D.H. Perkins, Ann. Rev. Nucl. Part. Sci. 34 (1984) 1.

[8] R. Barloutaud, Nucl. Phys. B (Proc. Suppl.) 28A (1992) 437. 
[9] Particle Data Group, Review of Particle Physics, Europ. Phys. J. C 15 (2000) 1.

[10] R. Bernabei et al., Phys. Lett. B 493 (2000) 12.

[11] G.N. Flerov et al., Sov. Phys. Dokl. 3 (1958) 79.

[12] Table of Isotopes, ed. by R.B. Firestone, V.S. Shirley et al., 8th ed. (John Wiley, New York, 1996).

[13] F.E. Dix, Ph. D. thesis, Case Western Reserve University, Cleveland, Ohio (1970).

[14] J.C. Evans, jr. and R.I. Steinberg, Science 197 (1977) 989.

[15] E.L. Fireman, Proc. Int. Conf. on Neutrino Phys. and Neutrino Astrophys. "Neutrino'77", Baksan Valley, USSR, 18-24 June 1977 (M., Nauka, 1978), v.1, p.53.

[16] R.I. Steinberg and J.C. Evans, Proc. Int. Conf. on Neutrino Phys. and Neutrino Astrophys. "Neutrino'77", Baksan Valley, USSR, 18-24 June 1977 (M., Nauka, 1978), v.2, p.321.

[17] S.P. Riley et al., Phys. Rev. C 59 (1999) 1780.

[18] H.H. Chen, Phys. Rev. Lett. 55 (1985) 1534;

J. Boger et al. (SNO Collaboration), Nucl. Instrum. Meth. A 449 (2000) 172.

[19] Yu.V. Kozlov et al., Phys. At. Nucl. 61 (1998) 1268; 63 (2000) 1016.

[20] J. Busenitz, Phys. At. Nucl. 63 (2000) 993.

[21] L.A. Mikaelyan, V.V. Sinev, Phys. At. Nucl. 63 (2000) 1002.

[22] G. Gratta, Nucl. Phys. B (Proc. Suppl.) 85 (2000) 72.

[23] Y. Declais, Nucl. Phys. B (Proc. Suppl.) 70 (1999) 148.

[24] B. Achkar et al., Nucl. Phys. B 434 (1995) 503.

[25] A. Piepke et al., Nucl. Instrum. Methods A 432 (1999) 392.

[26] M. Appolonio et al., Phys. Lett. B 420 (1998) 397.

[27] R.G.H. Robertson (for the SNO Collaboration), Prog. Part. Nucl. Phys. 40 (1998) 113. 